Ann. Biol. anim. Bioch. Biophys., Ig68, 8 (2), 209-216.

\title{
ANALYSE DES VARIATIONS SAISONNIÈRES DE LA FERTILITÉ DES BOVINS DANS LE JURA FRANÇAIS
}

\author{
M. COUROT, M. GOFFAUX ('), R. ORTAVANT \\ avec la collaboration technique de $R$. et M. Vuidepot $\left({ }^{2}\right)$ \\ Laboratoire de Physiologie de la Reproduction, \\ Centre de Recherches vétérinaires et zootechniques de Tours-l'Orfrasière, 37 - Nouzilly \\ Institut national de la Recherche agronomique
}

\section{SOMMAIRE}

La fertilité des bovins Montbéliard varie d'une façon cyclique au cours de l'année : élevée des mois d'octobre à février, elle atteint son niveau le plus bas au cours des mois de mai, juin et juillet. La diminution de la fertilité observée au printemps peut être attribuée aux vaches d'autant plus que le sperme possède à cette époque, une fécondance supérieure à celle qu'il a en automne.

\section{INTRODUCTION}

L'existence d'une variation à périodicité saisonnière dans les phénomènes de reproduction est bien connue chez certaines espèces animales domestiques telles que les ovins ou les caprins. Beaucoup moins marquée chez les bovins, elle existe cependant comme en témoignent les rapports récents sur ce sujet (SALISBURY et VANDEMARK, I96I ; BoDisCo et MAZZARI, I962; Poston, Ul_BERG et LEGATES, I962 ; STOTT et WILLIAMS, I962 ; KELI, et HURST, I963; OR'TAVANT, MaUlÉon et ThiBAULT, I964; VAN DUIJN, I964; ThrbaUlT et al., Ig66 ; SAlisbuRY, I967). Si l'on a mis en évidence une variation saisonnière de la fécondité bovine appréciée d'une façon globale par la fluctuation du taux de non-retours après insémination artificielle, les parts respectives de responsabilité des mâles et des femelles dans cette variation restent mal connues. Pour élucider ce problème, nous avons eu recours à une inversion expérimentale des saisons en inséminant les vaches au cours de la période de haute fécondité naturelle avec du sperme récolté et congelé en période de basse fécondité et inversement. Ce sont les résultats de ce travail que nous rapportons.

(1) U. N. C. E. I. A., 28, rue Bayard, Paris.

(2) Coopérative d'Élevage, 39 - Crançot.

Annales de Biologie animale. - Ig68. 


\section{MATÉRIEL, ET MÉTHODES}

Ce travail a été réalisé dans le Jura, région partagée entre l'élevage de plaine et l'élevage de montagne, où les bovins sont en stabulation permanente de novembre à avril en général. Il s'est déroulé dans le cadre des activités de la Coopérative d'Insémination artificielle du département ( $\left.{ }^{1}\right)$ sur des bovins laitiers de la race Montbéliarde.

La variation naturelle de la fertilité au cours de l'année a été observée à partir des résultats d'insémination artificielle de 7 années successives (octobre 1957 à septembre 1964). Ils portent sur 326874 inséminations premières réalisées avec le sperme de I 20 taureaux environ. Toutes ces inséminations ont été faites avec du sperme récolté chaque jour, conservé à $4^{\circ} \mathrm{C}$ et utilisé pendant 24 heures seulement. Les éjaculats étaient soumis aux examens habituels de laboratoire (volume, concentration, motilité.) puis dilués d'après leur concentration initiale de façon à obtenir 20 ou $3^{\prime} \cdot{ }^{\prime} 0^{\prime}$ spermatozol̈des par dose de I $\mathrm{ml}$ de semence; différents dilueurs ont été successivement employés : citrate-jaune d'ouf, laiciphos, lait, et enfin CU I6.

La partie expérimentale de ce travail a duré un an et demi (du printemps I964 à l'automne 1965). Treize taureaux dont le sperme supportait les techniques de congélation ont été soumis à une récolte par semaine. Un éjaculat a été congelé toutes les deux semaines, suivant une technique déjà décrite (GOFFAUX, I966), au cours du printemps Ig64 ( $2^{\text {e }}$ quinzaine de mars à I $^{\text {re }}$ quinzaine de juin) et de l'automne ig64 (octobre à décembre) ce qui a permis d'obtenir 68 et 64 éjaculats utilisables.

TABLEAU I

Protocole expérimental d'analyse des variations saisonnières de la fertilité bovine

\begin{tabular}{|c|c|c|c|}
\hline \begin{tabular}{l}
\multicolumn{2}{c}{ Récoite et traitement } \\
de la semence $\rightarrow$ \\
Circonstances \\
d'utilisation \\
de la semence \\
$\downarrow$ \\
\end{tabular} & $\begin{array}{l}\text { Sperme congelé } \\
\text { au printemps } 1964 \\
(15-3 \text { au } 15-6)\end{array}$ & $\begin{array}{l}\text { Sperme congelé } \\
\text { en automne 1964 } \\
(1 \text { er } 10 \text { au 31-12) }\end{array}$ & 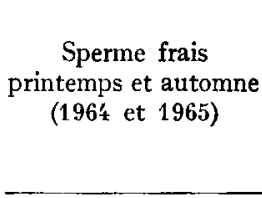 \\
\hline $\begin{array}{l}\qquad \text { Printemps } 1964 \\
\text { Nombre d'inséminations.......... } \\
\text { Conservation du sperme........ } \\
\text { Conditions d'utilisation........ } \\
\qquad \text { Automne } 1964\end{array}$ & $\begin{array}{c}3333 \\
0 \\
\text { en saison }\end{array}$ & & $\begin{array}{c}19005 \\
0 \\
\text { en saison }\end{array}$ \\
\hline $\begin{array}{l}\text { Nombre d'inséminations.......... } \\
\text { Conservation du sperme......... } \\
\text { Conditions d'utilisation......... } \\
\qquad \text { Printemps } 1965\end{array}$ & $\begin{array}{c}2388 \\
6 \text { mois } \\
\text { contre saison }\end{array}$ & $\begin{array}{c}2343 \\
0 \\
\text { en saison }\end{array}$ & $\begin{array}{c}11382 \\
0 \\
\text { en saison }\end{array}$ \\
\hline $\begin{array}{l}\text { Nombre d'inséminations.......... } \\
\text { Conservation du sperme........ } \\
\text { Conditions d'utilisation......... } \\
\qquad \text { Automne } 1965\end{array}$ & $\begin{array}{l}3491 \\
12 \text { mois } \\
\text { en saison }\end{array}$ & $\begin{array}{c}3080 \\
6 \text { mois } \\
\text { contre saison }\end{array}$ & $\begin{array}{c}11819 \\
0 \\
\text { en saison }\end{array}$ \\
\hline $\begin{array}{l}\text { Nombre d'inséminations.......... } \\
\text { Conservation du sperme........ } \\
\text { Conditions d'utilisation......... }\end{array}$ & & $\begin{array}{l}3108 \\
12 \text { mois } \\
\text { en saison }\end{array}$ & $\begin{array}{c}356 x \\
0 \\
\text { en saison }\end{array}$ \\
\hline
\end{tabular}

Après congélation, chaque éjaculat a été subdivisé en trois parties (tabl. r) destinées aux inséminations pratiquées respectivement dans la quinzaine qui a suivi la récolte (temps 0 , "en saison "), six mois après (" contre-saison ") et un an plus tard ("en saison "). Chaque fraction d'éjaculat a été utilisée pour l'insémination d'au moins 20 vaches (45 en moyenne) suivant un protocole précis de

(1) Nous tenons à remercier l'ensemble du personnel de cet organisme qui a très efficacement contribué à la realisation de cette expérience. 
distribution de la semence entre les différentes régions du département, établi pour éviter un biais des résultats ; 17743 inséminations premières ont ainsi été réalisées. En même temps, l'utilisation classique du sperme frais était poursuivie sur 45770 inséminations premières suivant la technique habituelle du centre, pour servir de référence.

Les résultats sont exprimés en pourcentage de non-retours, 3-6 semaines et 6o-9o jours après insémination. Leur signification statistique est appréciée par le test de $\chi^{2}$.

\section{RÉSULTATS}

\section{Variations saisonnières naturelles}

L,es données indiquant la variation moyenne habituelle observée sont présentées dans le tableau 2 . On remarque qu'après une période de haute fertilité, d'octobre à février $(7 \mathrm{I}, 3 \mathrm{p}$. Ioo $\mathrm{NR}$, avec maximum en novembre), une diminution régulière

TABLEAU 2

Variations mensuelles des non-retours après insémination artificielle des bovins dans le Jura (1)

\begin{tabular}{|c|c|c|c|c|}
\hline & $\begin{array}{l}\text { Inséminations } \\
\text { premières }\end{array}$ & $\begin{array}{c}\text { Non-retours à } \\
\text { 3-6 semaines (\%) }\end{array}$ & $\begin{array}{c}\text { Non-retours à } \\
60-90 \text { jours }(\%)\end{array}$ & $\begin{array}{l}\text { Différence non-retours } \\
\text { 3-6 sem. et } 60-90 \text { j } \\
\text { (Unités \%) }\end{array}$ \\
\hline Janvier .... & 28089 & 78,89 & 70,89 & 8,00 \\
\hline Février............. & 31305 & 79,04 & 70,77 & 8,27 \\
\hline Mars ......... & 34612 & 78,04 & 68,56 & 9,48 \\
\hline Avril................. & 40552 & 76,42 & 67,88 & 8,54 \\
\hline Mai ................. & 47943 & 73,04 & 65,48 & 7,56 \\
\hline Juin................ & 32996 & 73,03 & 65,03 & 8,00 \\
\hline Juillet ............ & 25649 & 73,02 & $64,91 *$ & 8,11 \\
\hline Aout............ & 19054 & -* & 68,47 & - \\
\hline Septembre .......... & $8716 *$ & 78,37 & 68,41 & 9,96 \\
\hline Octobre............. & $20 ; 37$ & 78,16 & 71,08 & 7,08 \\
\hline Novembre $\ldots \ldots \ldots \ldots$ & 17334 & 81,21 & 73,28 & 7,93 \\
\hline Décembre $\ldots \ldots \ldots \ldots$ & $198: 17$ & 79,19 & 71,01 & 8,18 \\
\hline Total $\ldots \ldots \ldots \ldots \ldots$ & 326874 & & & \\
\hline Moyennes $\ldots \ldots \ldots \ldots$ & & 76,54 & 68,34 & \\
\hline Octobre à février .... & 117352 & 79,19 & 71,27 & 7,92 \\
\hline Mai à juillet ........ & 106588 & 73,03 & 65,21 & 7,82 \\
\hline
\end{tabular}

(1) Octobre 1957 à septembre 1964 .

* Le nombre des données est relativement faible en septembre car le Centre d'Insémination artificielle cessait son activité pendant une partie de ce mois (vacances). C'est d'ailleurs pourquoi il n'y a pas de résultats à 3-6 semaines pour août et les résultats de juillet sont exprimés à 90-120 jours et non 60-90 jours.

commence en mars (fig. I) ; la différence entre mars et février est statistiquement significative. Cette diminution aboutit à un palier de basse fertilité de mai à juillet $(65,2$ p. Ioo NR) suivi d'une reprise au mois d'août. La différence du niveau des résultats entre les périodes d'automne et de printemps est très hautement significative. L'observation des courbes correspondant à chacune des sept années d'enregistrement 
de données montre que le même type de variation se retrouve régulièrement d'une année à l'autre, mais son importance relative est en fonction inverse du niveau général de fertilité de l'année considérée.

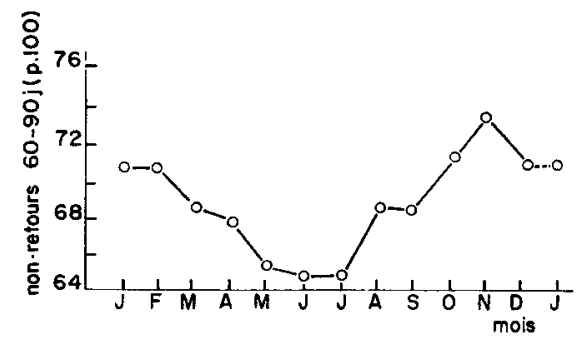

FIG. I. - Variations saisonnières de la fertilité bovine dans le Jura

Les taux de non-retours à 3-6 semaines et à 60-9o jours évoluent sensiblement de la même façon (tabl. 2) : la différence mensuelle entre les deux pourcentages est presque constante ( 8 unités de pourcentage environ) avec cependant deux pointes significatives en mars et en septembre, donc situées entre les périodes de haute et basse fertilité pour lesquelles cette différence est la même $(7,92 \mathrm{p}$. roo contre 7,82 p. roo).

\section{TABLEAU 3}

Influence respective des saisons de congélation et d'utilisation du sperme sur le taux de non-retours à 60-90 jours

\begin{tabular}{|c|c|c|c|}
\hline \multirow{2}{*}{ Insémination } & \multicolumn{3}{|c|}{ Congélation } \\
\hline & Printemps & Automne & Moyennes pondérées \\
\hline Printemps & $\begin{array}{l}61,4 \% \\
(6824)\end{array}$ & $\begin{array}{r}60,4 \% \\
(3080)\end{array}$ & $\begin{array}{l}61,1 \% \\
(9904)\end{array}$ \\
\hline Automne & $\begin{array}{l}68,0 \% \\
(2388)\end{array}$ & $\begin{array}{c}65,6 \% \\
(5451)\end{array}$ & $\begin{array}{c}66,4 \% \\
(7839)\end{array}$ \\
\hline Moyennes pondérées & $\begin{array}{c}63,1 \% \\
(9212)\end{array}$ & $\begin{array}{c}63,7 \% \\
(8531)\end{array}$ & $\begin{array}{c}63,4 \% \\
(17743)\end{array}$ \\
\hline
\end{tabular}

( ) Nombre d'inséminations premières.

Niveau de signification des résultats :

- Influence de la période de récolte $: a$ et $b, g$ et $h: \mathrm{NS}, d$ et $e: \mathrm{P} \leqslant 0,05$.

- Influence de la période d'insémination : $a$ et $d, b$ et $e, c$ et $f: \dot{\mathbf{P}} \leqslant \mathbf{0 , 0 1}$.

\section{Inversion expérimentale des saisons}

Les résultats de cette expérience sont présentés dans le tableau 3 et la figure 2 . Les inséminations de référence réalisées avec dus sperme frais (fig. 2) présentent la même variation saisonnière que celle analysée précédemment. L'existence de diffé- 
rences importantes entre les lots expérimentaux permet d'éclaircir l'origine de ces variations saisonnières : les résultats d'ensemble du tableau 3 montrent que, globalement, la saison de récolte du sperme semble être moins importante que la saison au cours de laquelle ce sperme est utilisé, c'est-à-dire la saison d'insémination. Cette dernière influe beaucoup sur le taux de réussite (6I,I p. Ioo NR à 60-90 jours au printemps contre $66,4 \mathrm{p}$. Ioo NR à 60-90 jours en automne ; la différence entre ces pourcentages moyens pondérés est très hautement significative). L'analyse des résultats détaillés apporte une illustration plus précise de ces phénomènes (fig. 2). Le sperme congelé au printemps possède un très bon potentiel fécondant puisqu'en dépit des résultats médiocres qu'il donne en cette saison (utilisé immédiatement - 6o,8 p. Ioo NR à 60-9o jours - ou un an plus tard - 62,0 p. Ioo NR), il permet d'obtenir d'excellents résultats lorsqu'il est utilisé en automne - 68,0 p. Ioo NR à 60-9o jours. Les résultats obtenus avec le sperme récolté en automne ont la même signification : 64,7 et 66,3 p. roo NR à $60-90$ jours en utilisation immédiate et un an plus tard, contre $60,4 \mathrm{p}$. Ioo NR à $60-90$ jours en utilisation de printemps. On peut donc en conclure que la responsabilité de la baisse de fertilité enregistrée au printemps est liée aux vaches.

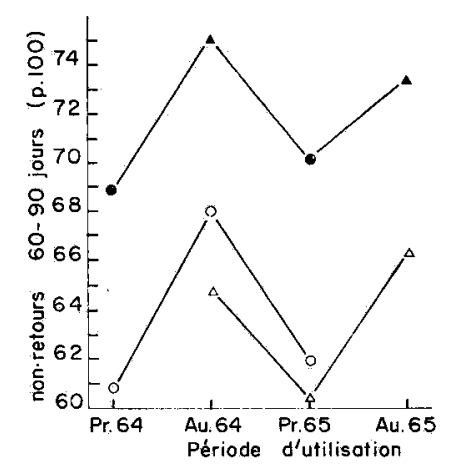

Fig. 2. - Influence de la période de récolte du sperme et de la période d'insémination sur la fertilité chez les bovins Sperme frais

- Récoltes de Printemps \ Récoltes d'Automne Sperme congelé

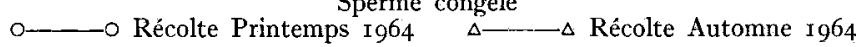

Cette évolution de la fertilité des vaches est plus importante que les chiffres ne le laissent paraître à première vue. Déjà les résultats d'ensemble (tab1. 3) groupés par saison d'utilisation montrent que le sperme de printemps possède une fécondance un peu supérieure à celle du sperme d'automne (mais ceci n'est significatif que pour les inséminations faites en automne $\mathrm{P} \leqslant 0.05$ ). Si pour l'analyse de l'effet " saison de récolte ", nous ne tenons compte que des inséminations réalisées au cours de la mếme période de temps avec les deux catégories de sperme (automne rg64 et printemps I965), nous observons une différence significative entre les pourcentages de non-retour en faveur du sperme de printemps $(64,4 \mathrm{p}$. Ioo contre $62,3 \mathrm{p}$. Ioo; $\mathrm{P} \leqslant 0,02)$. Cette différence de $2, \mathrm{I}$ unités de pourcentage peut être reliée aux critères classiques de qualité des éjaculats qui sont légèrement meilleurs au printemps (OrTAVANT, MAUL,́ion et Thrbault, I964; tabl. 4). Elle traduit une amélioration du pouvoir fécondant du 
sperme de printemps que masquent habituellement les variations inverses observées au niveau général des inséminations d'automne et de printemps.

TABLEAU 4

Caractéristiques des éjaculats congelés au printemps et en automne

\begin{tabular}{|c|c|c|}
\hline \multirow{2}{*}{ Caractéristiques du sperme } & \multicolumn{2}{|c|}{ Saison de récolte } \\
\hline & Printemps & Automne \\
\hline 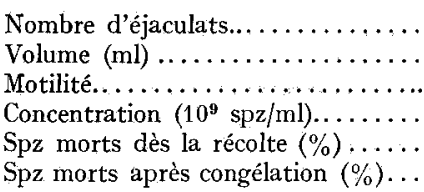 & $\begin{array}{l}68 \\
7,05 \\
4,31 \\
1,55 \\
22,41 \\
65,5\end{array}$ & $\begin{array}{l}64 \\
7,03 \\
4,29 \\
1,53 \\
26,71(\mathbf{1}) \\
69,0\end{array}$ \\
\hline
\end{tabular}

(1) Seule la différence entre ces deux valeurs est significative si l'analyse est faite à partir des valeurs moyennes de chaque Taureau.

\section{DISCUSSION}

Les revues récentes sur les variations saisonnières de la fertilité chez les bovins font apparaître une dispersion certaine des observations rapportées dans ce domaine. Différents auteurs en ont proposé un classement à partir duquel nous pouvons situer nos résultats (Salisbury et Van Demark, I96I ; OrTavanT, Mauléon et Thibaui,T, I964). Lorsqu'il existe une telle variation, elle est en général caractérisée par un à deux minima, un en été et l'autre en fin d'hiver et au printemps, ce dernier étant d'ailleurs assez discuté.

Le minimum d'été est le plus souvent observé dans les régions de latitude inférieure à $40^{\circ} \mathrm{N}$ (SAlisbURy et VAN DeMARK, I96I ; SAlisbURY, I967), où la température extérieure est élevée en cette période de l'année. On peut donc attribuer cette baisse temporaire de la fertilité à l'effet de la température, ce qui a d'ailleurs été démontré (KEILY et HuRST, I963; STOTTT et WilLIAMS, I962).

Le minimum de fin d'hiver et de printemps, comme celui observé dans le Jura, doit avoir une tout autre origine. Il ne peut pas être dû à la température, car les mois de mars, avril et mai où la baisse de fertilité commence à être observée, représentent une période encore tempérée $\left(t^{\circ}<20^{\circ} \mathrm{C}\right)$. SALISBURY et VAN DEMARK (I96I) ; ORTAVANT, MAULÉon et Thibault (I964), suggèrent un effet possible du photopériodisme. Nous devons alors admettre que ce phènomène n'agit que chez la vache, pour laquelle les conditions d'automne seraient les meilleures et qu'il reste sans effet chez le taureau car nous avons vu que seules les vaches étaient à l'origine de la chute de fertilité enregistrée au printemps. Une telle dissociation de l'effet du photopériodisme suivant le sexe a déjà été démontrée chez le lapin (WAITER, I967). Par 
ailleurs si la baisse de fertilité du printemps est souvent signalée en Europe (LEIDL, I953 ; VAN DUIJN, I964), elle n'a cependant pas le caractère d'universalité (SALIsBURY et VAN DEMARK, I96r) que l'on pourrait attendre des seuls effets des variations de la photopériode.

Sa situation en fin d'hiver et au printemps pourrait faire penser que cette diminution de fécondité a une origine nutritionnelle. Dans la région où nous avons travaillé, les vaches sont généralement en stabulation hivernale complète jusqu'au mois d'avril ; il faudrait alors admettre un effet résiduel prolongé du phénomène car 1'augmentation du pourcentage de non-retours n'apparaît qu'au mois d'août. Mais cette hypothèse d'une cause nutritionnelle permettrait de rendre compte de la différence des résultats observés entre mâles et femelles car les taureaux reçoivent une alimentation mieux contrôlée tout au long de l'année.

Une autre explication peut être proposée qui semble rendre mieux compte de la variation saisonnière de la fertilité : elle a trait au mode d'élevage des animaux. On remarque en effet que la période de plus faible fertilité correspond à celle où les animaux sont en pâture, condition qui permet une manifestation où une détection meilleures de l'œestrus. Ainsi la baisse du taux de réussite commence en mars, mois dont les retours à $60-90$ jours sont notés pendant le début de la sortie en pâture ; sa reprise intervient en août, mois dont les mêmes retours sont notés jusqu'en novembre (mois de stabulation). On comprendrait alors pourquoi seules les femelles sont en cause dans le phénomène observé. On pourrait aussi associer la variation de fertilité à un autre aspect de la conduite d'élevage qui tendrait à grouper les vêlages en début d'automne par une production de "lait d'hiver ». En réalité il n'en est rien car c'est au mois de mai que se situe le plus grand nombre de vaches inséminées dans cette région.

Mais, quelle qu'en soit la raison, la baisse de fertilité enregistrée au printemps, chez les vaches, affecte soit la fécondation de 1'œuf, soit les stades très précoces du développement embryonnaire puisque les écarts de non-retours à 3-6 semaines et 60-90 jours ne varient pas significativement avec les saisons ; ceci s'oppose aux résultats obtenus par les auteurs qui ont travaillé dans des régions de climat plus chaud (STOTT et Williams, I962; SALisbURy, I967).

De toutes manières, il est certain que cette variation saisonnière de la fertilité bovine est complexe. Son observation globale doit représenter le résultat de l'action de plusieurs facteurs dont le plus important peut d'ailleurs être différent suivant le sexe.

\section{CONCLUSION}

La variation annuelle de la fertilité des bovins observée dans une région de l'Est de la France est donc la résultante de deux variations d'amplitude inégale et de sens contraire : diminution importante de la fertilité des vaches et augmentation discrète de celle des taureaux au printemps.

A moins que la supériorité du pouvoir fécondant du sperme de printemps ne résulte d'un biais inconnu, la responsabilité du récepteur femelle dans la baisse globale de fertilité observée chez les bovins semble donc plus importante qu'on ne pouvait le prévoir. 


\section{SUMMARY}

SEASONAL VARIATIONS IN FERTILITY OF DAIRY CATTLE

A seven year study on fertility of Montbeliard cattle $(326,784$ first inseminations) points out the existence of a yearly peak-to peak fertility pattern (fig. I). Fertility reaches a maximum (71.3 per cent non-returns at 60-9o days) from October to February, then decreases in March to a minimum ( $P \leqslant 0.001 ; 65.2$ per cent non-returns at 60-90 days) in May, June and July, then increases again (table 2).

Experimental inversions of seasons between males and females, by autumn insemination of spring-frozen sperm and vice versa, show that cows are responsible for the decrease in spring fertility : 6r.I per cent non returns at 60-90 days for spring inseminations versus 66.4 per cent for autum. This is all the more conspicuous as the fertility power of spring sperm is 2.1 per cent higher than that of autumn sperm.

Neither temperature nor photoperiod consistently account for these phenomena.

\section{RÉFÉRENCES BIBLIOGRAPHIQUES}

Bodisco V. Y., Mazzarri G. B., 1962. Eficiencia reproductiva de las vacas Criollas y Pardo Suizas en el Centro de Investigaciones Agronomicas. Min. Agr. Ceniro Invest. Agr. Maracay, Vénezuela. Bol. Tecn., 14, pp. 24.

DuiJn C. VAN, 1964. Parameters of spermatozoan movement in relation to season of bulls in the Netherlands. V' Congr. Int. Reprod. anim., Trento, 4, 307-312.

GoFfaux M., ı966. Effets d'une durée de conservation de deux ans sur la motilité et le pouvoir fécondant du sperme de taureau. Elev. Ins., 92, 3-7.

Kelly J. W., Hurst V., I963. The effect of season on fertility of the dairy bull and the dairy cow. $J$. Am. Vet. Ass., 143, 40-43.

LEIDL W., I 958. Klima und sexual Funktion in männlicher Haustiere. M. Verlag und H. Schaper. Hanover, Germany.

Ortavant R., Mauleon P., Thibault C., 1964. Photoperiodic control of gonadal and hypophyseal activity in domestic mammals. Ann. N. Y. Acad. Sci., 117, 157-192.

Poston H. A., Ulberg L. C., Legates J. E., I962. Analysis of seasonal fluctuations of reproductive performance in dairy cows. J. Dairy, Sci., 45, 1376-1 379 .

Salisbury G. W., 1967. Aging phenomena in spermatozoa. III. Effect of season and storage at -79 to $-88^{\circ} \mathrm{C}$ on fertility and prenatal losses. J. Dairy Sci., 50, 1683-1689.

Salisbury G. W., Van DEmark N. L., I961. Physiology of reproduction and artificial insemination of cattle. Freeman and Co. ed.

Stotr G. H., Wrlliams R. J., 1962. Causes of low breeding efficiency in dairy cattle associated with seasonal high temperatures. J. Dairy Sci., 45, I $369^{-1} 375$.

Thibault C., Courot M., Martinet L., Mauléon P., du Mesnil du Buisson F., Ortavant R., Pelletier. J., Signoret J. P., I 966 . Regulation of breeding season and estrous cycles by light and external stimuli in some mammals. J. Anim. Sci., 25, Suppl., 1 19-142.

Walter M. R., 1967. Variation photopériodique et activité sexuelle du Lapin domestique. Thèse Doct. $3^{\circ}$ cycle, Paris. 